\title{
EVALUACIÓN DEL IMPACTO EN LA SALUD POR PARTÍCULAS PM 2.5 EN SINALOA, MÉXICO
}

Assessment of health impacts by $\mathrm{PM}_{2.5}$ particles in Sinaloa, Mexico

\author{
Luis Armando BECERRA PÉREZ y Roberto Alonso RAMOS ÁLVAREZ*
}

Facultad de Ciencias Económicas y Sociales, Universidad Autónoma de Sinaloa, Blvd. Universitarios y Av. de las Américas, Unidad 3 s/n, Ciudad Universitaria, 80010 Culiacán, Sinaloa, México

*Autor para correspondencia: robertoramos@uas.edu.mx

(Recibido: noviembre 2018; aceptado: septiembre 2019)

Palabras clave: contaminación ambiental, muertes evitables, pérdida de productividad, políticas ambientales

\section{RESUMEN}

Existe un creciente interés a nivel internacional por evaluar los efectos en la salud que genera la contaminación ambiental y su cuantificación económica. Este artículo analiza los impactos en la salud que causa la contaminación del aire por $\mathrm{PM}_{2.5}$ en las principales ciudades del estado de Sinaloa, México: Culiacán (capital), Mazatlán y Los Mochis, Ahome; asimismo, estima las muertes que se evitarían si la concentración de la contaminación se redujera a los niveles recomendados por la Organización Mundial de la Salud y la Norma Oficial Mexicana correspondiente, y calcula los costos económicos debidos a pérdida de productividad de las muertes asociadas a $\mathrm{PM}_{2.5}$. Se encontró que las muertes totales evitables serían 354 y 261 utilizando la norma internacional y mexicana, respectivamente. El costo económico calculado por exceder los límites permisibles de $\mathrm{PM}_{2.5}$ en las principales ciudades de Sinaloa estuvo en el rango de 24 a 34 millones de dólares para 2017. Se recomienda diseñar políticas ambientales locales que promuevan la reducción de los niveles de $\mathrm{PM}_{2.5}$, para lo cual se requiere identificar las emisiones por tipo de fuente con el objetivo de implementar acciones específicas que, desde una perspectiva local, contribuyan a la meta nacional de mitigar los impactos negativos de la contaminación del aire.

Key words: environmental pollution, avoidable deaths, loss of productivity, environmental policies

\begin{abstract}
There is a growing global interest in the evaluation of health effects generated by environmental pollution and its economic quantification. This article analyzes the health impacts caused by $\mathrm{PM}_{2.5}$ air pollution in the main cities of the state of Sinaloa, Mexico: Culiacán (capital), Mazatlán and Los Mochis, Ahome; it also estimates avoidable deaths if the concentration of contaminants was reduced to the levels recommended by the World Health Organization and the corresponding Official Mexican Standard, and monetizes the economic costs through the loss of productivity of associated deaths to $\mathrm{PM}_{2.5}$. It was found that avoidable total deaths would be 354 and 261 using the international and Mexican norms, respectively. The calculated economic cost of exceeding the permissible limits of $\mathrm{PM}_{2.5}$ in the main cities of Sinaloa was in the range of 24 to 34 million dollars for 2017. It is recommended to design local environmental policies
\end{abstract}


that promote the reduction of $\mathrm{PM}_{2.5}$ levels, for which it is necessary to identify emissions by source type with the objective of implementing specific actions that, from a local perspective, contribute to the national goal of mitigating the negative impacts of air pollution.

\section{INTRODUCCIÓN}

La mala calidad del aire debida al incremento en la emisión de contaminantes provoca graves daños en el sistema respiratorio y circulatorio del ser humano. Respirar aire contaminado es peligroso, como lo han demostrado múltiples investigaciones que relacionan los efectos de la contaminación del aire sobre la salud humana (Dockery y Pope 1994, INECC 2014, 2017, Alpízar et al. 2017, de Keijzer et al. 2017). Uno de los contaminantes más nocivos para la salud son las partículas de $2.5 \mu \mathrm{m}$ de diámetro $\left(\mathrm{PM}_{2.5}\right.$, por sus siglas en inglés).

De acuerdo con estudios epidemiológicos, las $\mathrm{PM}_{2.5}$ tienen efectos adversos sobre la salud, pudiendo ocasionar muertes prematuras que derivan de enfermedades como cardiopatía isquémica, accidentes cerebrovasculares, cáncer de pulmón, enfermedad pulmonar obstructiva crónica e infecciones respiratorias (Cohen et al. 2005, 2017, Pope et al. 2011, Lim et al. 2012, Apte et al. 2018).

Estos impactos sobre la salud están relacionados en términos de número de muertes y años de vida perdidos, los cuales se ven influenciados por la carga de este tipo de enfermedades (Forouzanfar et al. 2015).

Según la Organización Mundial de Salud (OMS), en 2016 a nivel global siete millones de muertes al año estuvieron vinculadas con la exposición a la contaminación del aire tanto de interiores como de exteriores (OMS 2016). De esos siete millones de fallecimientos según la Carga Global de Enfermedades (GBD, por sus siglas en inglés) se determinó que las $\mathrm{PM}_{2.5}$ fueron responsables de 4.1 millones (Naghavi et al. 2017, Gakidou et al. 2017), lo que representó aproximadamente el $7.1 \%$ de la mortalidad global (Evans et al. 2013).

De Keijzer et al. (2017) encontraron que un incremento de $5 \mu \mathrm{g} / \mathrm{m}^{3}$ en la concentración de $\mathrm{PM}_{10}$ se traducía en una pérdida de casi un año de vida en la ciudad de Barcelona, España. Además, estos mismos autores concluyeron que un aumento de $2 \mu \mathrm{g} / \mathrm{m}^{3}$ en la concentración de $\mathrm{PM}_{2.5}$ está relacionado con una reducción de siete meses de vida.

De igual forma, un análisis de tasas de mortalidad estandarizadas reveló que dichos incrementos en la concentración de $\mathrm{PM}_{10}$ y $\mathrm{PM}_{2.5}$ están asociados con un aumento del riesgo de mortalidad del $5.7 \%$ y del $3.7 \%$, respectivamente (de Keijzer et al. 2017).

Actualmente, China, Estados Unidos y Europa son las regiones del mundo más analizadas por la literatura especializada sobre impactos de la contaminación del aire y su evaluación económica, y se ha corroborado que esos países exceden tanto los límites permisibles de $\mathrm{PM}_{10}$ y $\mathrm{PM}_{2.5}$ establecidos por la OMS como los de su propia legislación ambiental. En los últimos años han aumentado los datos que evidencian el efecto adverso de la contaminación atmosférica y el incremento de las tasas de mortalidad por estos motivos (USEPA 2011, Pascal et al. 2013, Chanel et al. 2016, Wu et al. 2017).

En el caso de México, el Instituto Nacional de Ecología y Cambio Climático (INECC 2014, 2017) ha cuantificado los impactos atribuibles a la contaminación del aire. Por su parte, el Instituto Mexicano de la Competitividad (IMCO) realizó un estudio con una herramienta metodológica de su propia creación, que estima los costos de salud y la pérdida de productividad por muertes atribuibles a la contaminación en 34 ciudades mexicanas para 2010. Dicha herramienta determina los costos totales por contaminación de $\mathrm{PM}_{10}$ en México considerando gastos de salud y pérdidas de productividad, para lo cual utiliza: 1) un indicador $\beta$ de 0.0006 obtenido de la literatura epidemiológica, con el cual se obtuvo un resultado de 5690.6 millones de pesos mexicanos; y 2) un indicador $\beta$ de 0.0009 calculado por los investigadores del IMCO, con el cual se calculó un costo total de 4123.7 millones de pesos mexicanos.

Siguiendo la metodología antes mencionada, el objetivo de este artículo es analizar los impactos en la salud que causa la contaminación del aire por $\mathrm{PM}_{2.5}$ en las principales ciudades del estado de Sinaloa, México, determinando las muertes prematuras evitables y el monto económico por la pérdida de productividad que representan esas muertes. Existe evidencia de que los niveles de contaminación del aire por $\mathrm{PM}_{2.5}$ en las ciudades estudiadas rebasa los límites permitidos, lo cual se refleja en la pérdida de productividad que se genera y la cantidad de dinero que las personas de 14 a 65 años de edad dejaran de percibir al estar en riesgo de morir a causa de la contaminación por $\mathrm{PM}_{2.5}$. 
Es importante destacar que la mayoría de los estudios realizados en México se han concentrado en las tres principales zonas metropolitanas del país (Ciudad de México, Guadalajara y Monterrey), que dado los altos niveles de concentración de emisiones de los diferentes contaminantes $\left(\mathrm{CO}, \mathrm{CO}_{2}, \mathrm{NO}, \mathrm{NO}_{2}\right.$, $\mathrm{NO}_{\mathrm{X}}, \mathrm{O}_{3}, \mathrm{SO}_{2}, \mathrm{PM}$ ) y por el número de población expuesta, son urbes más atractivas para el análisis y sus resultados son más demandados por parte de las autoridades encargadas de vigilar y mitigar los efectos negativos de la contaminación. Sin embargo, existe un grupo importante de ciudades medias, como las aquí estudiadas, que requieren ser analizadas para valorar desde el punto de vista económico los impactos sobre la salud y, en consecuencia, diseñar políticas públicas locales que complementen el objetivo nacional de contrarrestar dichos efectos negativos sobre la población y el desempeño económico de las localidades.

El artículo está organizado en cuatro partes. La primera, la presente introducción, que enmarca la importancia de este análisis con base en la literatura reciente, indicando el objetivo de la investigación; la segunda describe la metodología utilizada, los datos y la zona de estudio; la tercera presenta los resultados del análisis empírico y su discusión; la cuarta proporciona las conclusiones del trabajo.

\section{MATERIALES Y MÉTODOS}

Esta investigación utiliza la metodología de evaluación económica del impacto en la salud por contaminación de $\mathrm{PM}_{2.5}$, que inicia con la definición del tamaño de la muestra, en este caso ciudades medias alrededor de 500000 habitantes con un sistema de monitoreo de la calidad del aire acorde con la NOM-156-SEMARNAT-2012 (SEMARNAT 2012) establecido y en operación. En segundo lugar, selecciona el contaminante a investigar, además de las variables en las que se basará el estudio, así como sus respectivas fuentes y año de observación. En tercer lugar considera un dato importante para estimar los impactos en salud atribuibles a la contaminación del aire, que son las funciones concentración-respuesta (FCR). Para determinar el riesgo relativo se hace una revisión exhaustiva de la literatura epidemiológica que, generalmente, presenta riesgos relativos (RR) ante un determinado cambio en la concentración (el cambio más común es de $10 \mu \mathrm{g} / \mathrm{m}^{3}$ ). En cuarto lugar, se calculan los impactos atribuibles a la contaminación del aire, que son los gastos en salud y la pérdida en productividad. En nuestro caso, únicamente se incluyó la pérdida de productividad y se usó información geográfica así como de población, muertes, salarios, inflación y concentración de contaminantes proveniente únicamente de fuentes oficiales, como el Instituto Nacional de Estadística y Geografía (INEGI), el Banco de México (BANXI$\mathrm{CO}$ ), el Consejo Nacional de Población (CONAPO) y el Gobierno del Estado de Sinaloa.

Para llevar a cabo el análisis empírico de esta investigación, se utilizó una herramienta desarrollada por el Instituto Mexicano de Competitividad (IMCO 2013) que tiene dos ventajas: primero, asocia los impactos en la salud con las pérdidas económicas que éstos implican; segundo, presenta indicadores desagregados por localidad, lo cual facilita el análisis de zonas urbanas específicas. La calculadora de impactos por contaminación del aire es una herramienta flexible y versátil que permite utilizar varios supuestos y parámetros para estimar los costos que genera este problema ambiental en las ciudades mexicanas, donde la limitante real no es metodológica sino los datos de concentración de contaminantes, salarios, hospitalizaciones y muertes, entre otros, ya que a medida que se avanza en la desagregación de la población objetivo y el registro detallado de causas de hospitalización/muerte, el grado de dificultad se incrementa gradualmente.

\section{Área y sujetos de estudio}

El estado de Sinaloa se ubica en el noroeste de México. S us ciudades más importantes son Culiacán, Mazatlán y Los Mochis (Ahome), que en forma conjunta concentran el $63 \%$ de la población total de la entidad (Fig. 1). La ciudad de Culiacán, capital del estado, se ubica entre los paralelos $24^{\circ} 02^{\prime}$ y $25^{\circ} 17^{\prime}$ de latitud norte, y los meridianos $106^{\circ} 52^{\prime}$ y $107^{\circ} 50^{\prime}$ de longitud oeste. Es una zona urbanizada que cuenta con una población de 957613 habitantes (31.6\% del total estatal), una densidad poblacional de $143.6 \mathrm{hab} / \mathrm{km}^{2}$ y una temperatura de 18 a $26{ }^{\circ} \mathrm{C}$. La ciudad de Mazatlán, municipio del mismo nombre, se ubica entre los paralelos $23^{\circ} 04^{\prime}$ y $23^{\circ} 54^{\prime}$ de latitud norte, y los meridianos $105^{\circ} 55^{\prime}$ y $106^{\circ}$ $38^{\prime}$ de longitud oeste. Es una zona urbanizada que cuenta con una población de 488281 habitantes (el $16.1 \%$ del total estatal), una densidad poblacional de $198.5 \mathrm{hab} / \mathrm{km}^{2}$ y una temperatura de 14 a $26{ }^{\circ} \mathrm{C}$. La ciudad de Los Mochis, municipio de Ahome, se ubica entre los paralelos $25^{\circ} 26^{\prime}$ y $26^{\circ} 25^{\prime}$ de latitud norte, y los meridianos $108^{\circ} 45^{\prime}$ y $109^{\circ} 28^{\prime}$ de longitud oeste. Es una zona urbanizada que cuenta con una población de 459873 habitantes (el 15.3\% del total estatal), una densidad poblacional de $112.4 \mathrm{hab} / \mathrm{km}^{2}$ y una temperatura de 22 a $26^{\circ} \mathrm{C}$ (INEGI 2010, 2015, 2017). 


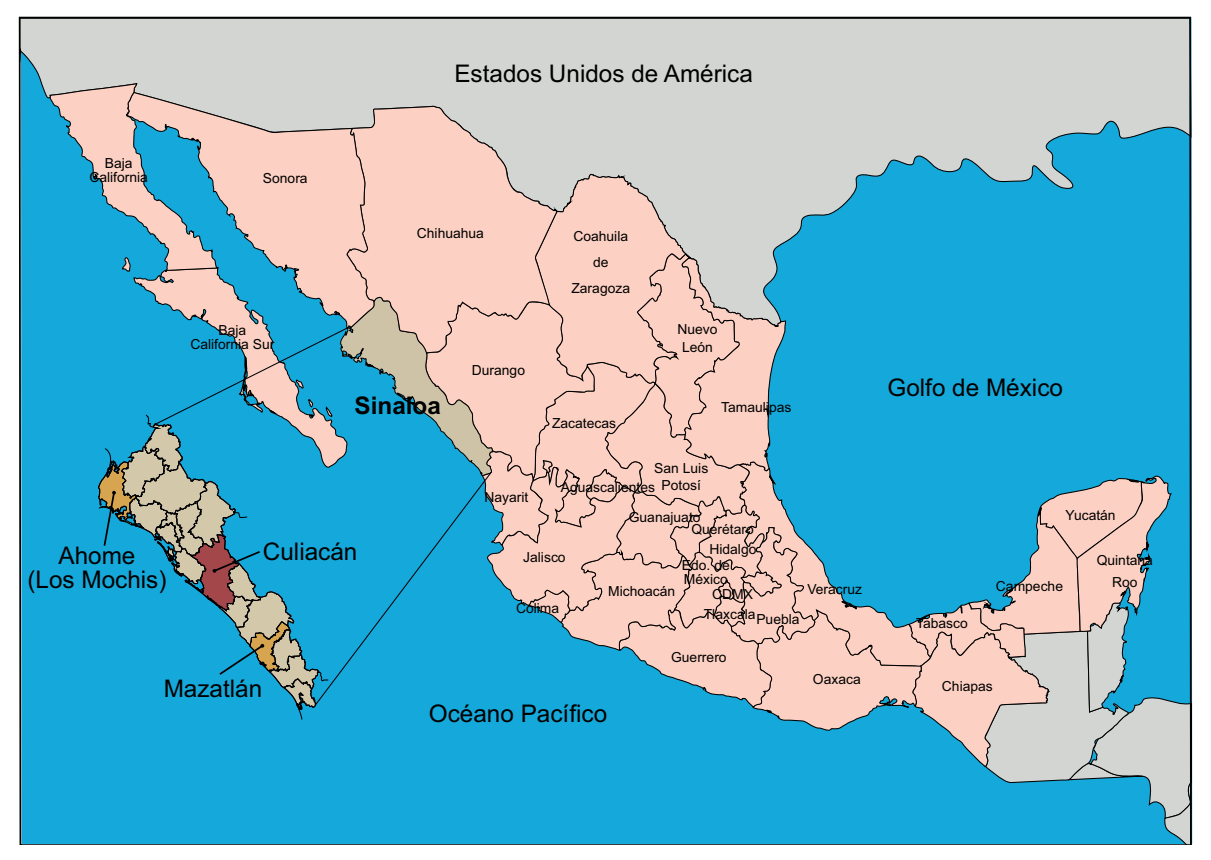

Fig. 1. Sinaloa es un estado de México que se localiza al noroeste del país; se ubica entre los paralelos $27^{\circ} 02^{\prime} 32^{\prime \prime}$ y $22^{\circ} 28^{\prime} 02^{\prime \prime}$ de latitud norte, y los meridianos $105^{\circ} 23^{\prime} 32^{\prime \prime}$ y $109^{\circ} 26^{\prime}$ 52 " de longitud oeste, con una superficie de $57377.2 \mathrm{~km}^{2}$. Sus tres principales ciudades son Culiacán (capital), Mazatlán y Los Mochis. Fuente: INEGI 2010, SAGARPA 2017

Las principales fuentes de contaminación atmosférica fijas en Sinaloa son las actividades agroindustriales, la minería y la generación de electricidad (cuenta con dos termoeléctricas, una en Mazatlán y otra en Ahome), mientras que las fuentes de contaminación móviles son los vehículos motores, incendios forestales y quemas agrícolas.

Para esta investigación se tomó en cuenta a una población expuesta al contaminante de 14 a 65 años de edad, residente en las ciudades de Culiacán, Mazatlán y Los Mochis durante el año de estudio (2017). El rango de edades seleccionado obedece a que se considera como la etapa productiva en países en desarrollo en la literatura de evaluación económica sobre el impacto en la salud (Rice 1985, Chang et al. 2016).

\section{Selección del contaminante}

$\mathrm{El}$ análisis selecciona a las $\mathrm{PM}_{2.5}$ como indicador de la contaminación del aire. Típicamente este contaminante tiene como fuente principal los motores de combustión interna (automóviles), actividades industriales, generación de electricidad, minas, resuspensión de polvo, quemas agrícolas y algunos factores naturales como incendios forestales y emisiones volcánicas.

Este tipo de partículas, mientras más pequeñas, más fácilmente pueden introducirse en las vías respiratorias hasta llegar a los pulmones, donde se alojan en los alvéolos. Se han asociado con padecimientos respiratorios y cardiovasculares, llevando a algunos pacientes hasta la muerte (Rojas-Bracho y Garibay-Bravo 2003). Las PM 2.5 usualmente incluyen en su composición algunos químicos altamente dañinos para la salud humana.

\section{Datos}

Los datos principales para esta investigación se obtuvieron de las concentraciones anuales promedio de $\mathrm{PM}_{2.5}$ correspondientes a 2017 de las estaciones de monitoreo de Culiacán, Mazatlán y Los Mochis, Ahome, los cuales fueron proporcionados por la Secretaría de Medio Ambiente del Gobierno del Estado de Sinaloa. Los datos de población de cada una de las áreas estudiadas fueron tomados del Censo de Población y Vivienda 2010, realizado por el INEGI y actualizado por el CONAPO para 2017. Las estadísticas de muertes totales de las tres ciudades, excluyendo las accidentales y violentas, fueron obtenidas del Sistema Estatal y Municipal de Bases de Datos (SIMBAD). Todas estas variables las hemos concentrado en el cuadro I por fuente y año.

Además, el estudio requirió de los parámetros de concentración objetivo anual promedio de $\mathrm{PM}_{2.5}$ recomendados por la OMS (2006) y la Norma Oficial 
CUADRO I. PARÁMETROS Y VARIABLES SOCIALES UTILIZADAS

\begin{tabular}{llc}
\hline Variable & Fuente & Año \\
\hline Población (censo) & INEGI (2010) & 2010 \\
Población proyectada & CONAPO (2017) & 2017 \\
\hline PM $_{2.5}$ (concentración promedio anual) & $\begin{array}{l}\text { Sistemas de monitoreo del Gobierno del Estado de Sinaloa, } \\
\text { procesados de acuerdo con las especificaciones de la NOM- }\end{array}$ & 2017 \\
& 025-SSA1-2014 <incluir esta fuente en las referencias $>$ & \\
\hline $\begin{array}{l}\text { Muertes totales (excluyendo } \\
\text { accidentales y violentas) }\end{array}$ & INEGI (2017a) & 2017 \\
\hline
\end{tabular}

Fuente: elaboración propia a partir de datos del INEGI (2010, 2017) y CONAPO (2017)

Mexicana NOM-025-SSA1-2014 (SSA 2014), los cuales son 10 y $12 \mu \mathrm{g} / \mathrm{m}^{3}$, respectivamente. Estas concentraciones objetivo deben interpretarse como los valores máximos permisibles para que los impactos en la salud de las personas expuestas a $\mathrm{PM}_{2.5}$ sean nulos. Por último, el salario promedio diario fue estimado a partir de la Encuesta Nacional de Ingresos y Gastos de los Hogares (ENIGH) (INEGI 2016). Estas variables se presentan en el cuadro II.

CUADRO II. PARÁMETROS Y VARIABLES ECONÓMICAS UTILIZADAS

\begin{tabular}{lcc}
\hline Variable & Fuente & Año \\
\hline $\mathrm{PM}_{2.5}$ (concentración promedio anual): & & \\
$10 \mu \mathrm{g} / \mathrm{m}^{3}$ & OMS & 2006 \\
$\mathrm{PM}_{2.5}($ concentración promedio anual): & SSA & 2014 \\
$12 \mu \mathrm{g} / \mathrm{m}^{3}$ & INEGI & $2017 \mathrm{~b}$ \\
\hline Salario diario: $\$ 556^{*}$ & & \\
Salario anual: $\$ 202940^{*}$ & & \\
\hline
\end{tabular}

*Actualizado a 2017 con el Índice Nacional de Precios al Consumidor (INPC), INEGI (2017b)

Fuente: elaboración propia a partir de datos de la OMS, SSA e INEGI

\section{Funciones concentración-respuesta y riesgo re- lativo}

De acuerdo con el Instituto Nacional de Ecología de la Secretaría de Medio Ambiente y Recursos Naturales (INE 2011), las funciones concentraciónrespuesta (FCR) son el componente más importante para estimar los impactos en salud atribuibles a la contaminación del aire. Éstas representan la probabilidad de que ocurra un impacto en salud a partir de un cambio en la concentración de un contaminante al que está expuesta la población. En otras palabras, estas funciones determinan qué tan sensible es la población a padecer efectos negativos a causa de la contaminación.

Las FCR son resultado de estudios epidemiológicos que se encuentran expresados en la literatura en términos de riesgo relativo (RR). Cuando el período es largo, se prefieren las FCR de estudios de cohorte. En México no se cuenta con estudios de cohorte, por lo que se tiene que recurrir a estudios a nivel internacional. Existen dos estudios pioneros en Estados Unidos que muestran datos sobre riesgo relativo asociado con la mortalidad por diversas enfermedades cardiovasculares y por cáncer de pulmón. El estudio de la Sociedad Americana del Cáncer (ACS, por sus siglas en inglés) y el estudio de seis ciudades (Dockery et al. 1993).

Los estudios de serie de tiempo muestran los impactos a corto plazo de variaciones de la contaminación atmosférica (OMS 2001, Hurley et al. 2005). El metaanálisis epidemiológico usado en esta investigación considera los efectos por mortalidad general, tomando como base un estudio de Hoek et al. (2013) $\mathrm{y}$ analizando el riego relativo de las concentraciones promedio de $\mathrm{PM}_{2.5}$. Dada la escasez de datos sobre el RR para México y, específicamente para las tres ciudades en estudio, se buscaron alternativas en la literatura epidemiológica, y se encontró que el cambio más común utilizado es de $10 \mu \mathrm{g} / \mathrm{m}^{3}$. Sin embargo, para capturar mejor los impactos atribuibles a la contaminación utilizando diferentes cambios en la concentración, es necesario encontrar el estimador $\beta$ en el que se basa el RR, para lo cual se utiliza la siguiente ecuación:

$\beta=\frac{\ln (R R)}{\Delta \operatorname{conc}}$ 
donde $\beta$ es el estimador, $\ln (R R)$ es el logaritmo natural del riesgo relativo $(R R)$, y Dconc es el cambio en la concentración que corresponde al RR reportado. Así, $\beta$ captura la probabilidad de que ocurra un evento, en nuestro caso enfermar o morir. El $R R$ mide la fuerza de asociación entre la exposición y el evento, en individuos expuestos y no expuestos a un factor de riesgo estimado a partir de los grupos de estudio (Morris y Gardner 1988). El $\beta$ utilizado en la presente investigación fue calculado por Krewski et al. (2009) tomando en cuenta un $R R$ de 1.06 de la FCR que siguen usando Hoek et al. (2013), y el valor medio que encontraron fue de (0.006) que es el más usado para $\mathrm{PM}_{2.5}$. El cuadro III muestra los estimadores empleados por Krewski et al. (2009) para calcular el valor medio de $\beta$ tomado para este estudio, incluyendo la fuente y el intervalo de confianza. Por su parte, el cuadro IV muestra los $\beta$ de concentración del contaminante analizado. En este estudio se tomó el valor de la media.

\section{RESULTADOS Y DISCUSIÓN}

Los primeros resultados se muestran en el cuadro $\mathbf{V}$, donde se comparan las concentraciones promedio anual de $\mathrm{PM}_{2.5}$ de las ciudades de Culiacán, Mazatlán y Los Mochis, Ahome, vs. la concentración objetivo indicada por las normas OMS (internacional) y NOM-025-SSA1-2014 (mexicana).

Se confirma que la concentración promedio anual de $\mathrm{PM}_{2.5}$ de las tres principales ciudades de Sinaloa está por arriba de la concentración objetivo de las normas internacional y mexicana, por lo que es necesario reducir las concentraciones de dicho contaminante. Culiacán excede la norma internacional en $32 \%$ y la norma mexicana en $10 \%$, Mazatlán sobrepasa la norma internacional en $122 \%$ y la norma mexicana en $85 \%$, y Los Mochis, Ahome, rebasa la norma internacional en $100 \%$ y la norma mexicana en $67 \%$.

Desde este simple punto de vista, la ciudad que excede en mayor medida los límites permisibles del

CUADRO III. ESTIMADORES DE LA LITERATURA EPIDEMIOLÓGICA

\begin{tabular}{|c|c|c|c|c|}
\hline Impacto en salud & $\begin{array}{c}\text { Causas (Catálogo Internacional } \\
\text { de Enfermedades-10) }\end{array}$ & $\begin{array}{l}\text { Grupo de } \\
\text { edad }\end{array}$ & $\begin{array}{c}{\text { (Riesgo relativo })^{2} \text { Función }} \\
\text { concentración respuesta } \\
(95 \%) \mathrm{PM}_{2.5}\end{array}$ & Fuente \\
\hline $\begin{array}{l}\text { Muertes prematuras } \\
\text { por todas las causas }\end{array}$ & (A00-R99) & $\geq 14$ años & $1.06(1.04-1.08)$ & Hoek et al. (2013) \\
\hline
\end{tabular}

${ }^{1}$ Clasificación Internacional de Enfermedades y Muertes de la OMS; ${ }^{2}$ riesgo relativo, implica la probabilidad de ocurrencia de un evento, enfermar o morir, en individuos expuestos y no expuestos a un factor de riesgo estimado a partir de los grupos de estudio; ${ }^{3}$ función concentración-respuesta al $95 \%$ de confianza

Fuente: elaboración propia a partir de Hoek et al. 2013

CUADRO IV. $\beta$ DE CONCENTRACIÓN DE ACUERDO CON LA LITERATURA

\begin{tabular}{lcccc}
\hline Impacto en salud & Media & Límite inferior & Límite superior & Fuente \\
\hline $\begin{array}{l}\text { Muertes prematuras } \\
\text { por todas las causas }\end{array}$ & 0.006 & 0.0007 & 0.012 & Krewski et al. (2009) \\
\hline
\end{tabular}

Fuente: elaboración propia a partir de la metodología usada por Krewski et al. (2009)

CUADRO V. CONCENTRACIÓN PROMEDIO DE PM 2.5 EN CULIACÁN, MAZATLÁN Y LOS MOCHIS, Y CONCENTRACIÓN OBJETIVO DE LA NORMA OMS Y LA NORMA OFICIAL MEXICANA NOM-025-SSA1-2014

\begin{tabular}{lccc}
\hline Ciudad & $\begin{array}{c}\text { Concentración anual } \\
\text { promedio }\left(\mu \mathrm{g} / \mathrm{m}^{3}\right)\end{array}$ & $\begin{array}{c}\text { Concentración objetivo } \\
\text { internacional }\left(\mu \mathrm{g} / \mathrm{m}^{3}\right)\end{array}$ & $\begin{array}{c}\text { Concentración } \\
\text { objetivo mexicana }\left(\mu \mathrm{g} / \mathrm{m}^{3}\right)\end{array}$ \\
\hline Culiacán & 13.21 & 10 & 12 \\
Mazatlán & 22.20 & 10 & 12 \\
Los Mochis & 20.06 & 10 & 12 \\
\hline
\end{tabular}

Fuente: elaboración propia a partir de datos de la OMS y NOM-025-SSA1-2014 
contaminante $\mathrm{PM}_{2.5}$ es Mazatlán, lo que nos lleva a inferir que es la zona urbana de Sinaloa que más podría impactar la salud de sus residentes, situación que contrasta con la definición de ciudad turística internacional (industria limpia) y su proyección como puerto comercial.

Los cuadros VI y VII exponen los resultados de muertes que podrían evitarse si se redujeran los niveles de $\mathrm{PM}_{2.5}$ a los niveles indicados por la norma internacional y la norma mexicana. Estos cuadros también muestran estimaciones económicas por la pérdida de productividad asociada a dichas muertes, que pueden interpretarse como el costo monetario para la sociedad ocasionada por exceder los límites permisibles de $\mathrm{PM}_{2.5}$. Dichas muertes podrían prevenirse mediante políticas públicas ambientales que obliguen a las fuentes a mantener sus emisiones dentro de los límites establecidos por la norma.

Las estimaciones realizadas muestran que en total 354 muertes por año podrían ser evitadas si las tres ciudades principales de Sinaloa lograran bajar sus emisiones actuales de $\mathrm{PM}_{2.5}$ a un nivel de $10 \mu \mathrm{g} / \mathrm{m}^{3}$ (Cuadro VI). La ciudad de Mazatlán evitaría 171,
Los Mochis 113 y Culiacán 70 muertes al año. El costo de estos impactos a la salud pública, monetizadas por la pérdida de productividad que se genera en la economía local a raíz de esas muertes, se estima en 645.1 millones de pesos mexicanos. Utilizando un tipo de cambio promedio de 18.90 pesos/USD (BANXICO 2018), la pérdida económica (costo) por exceder el límite de este contaminante en las tres principales ciudades de Sinaloa fue de aproximadamente 34.1 millones de USD en el año 2017.

El cuadro VII muestra las estimaciones obtenidas mediante la aplicación de la norma ambiental mexicna NOM-025-SSA1-2014, la cual marca un límite $2 \mu \mathrm{g} / \mathrm{m}^{3}$ mayor que la norma internacional (OMS). De forma similar, los resultados muestran que la ciudad con mayores impactos en la salud es Mazatlán y la de menores impactos es Culiacán. En este caso, se evitarían en total 261 muertes si los niveles de emisiones de $\mathrm{PM}_{2.5}$ se redujeran a $12 \mu \mathrm{g} / \mathrm{m}^{3}$, mientras que las pérdidas de productividad se calculn en 464.7 millones de pesos mexicanos, equivalentes a 24.6 millones de dólares estadounidenses para 2017.

CUADRO VI. MUERTES EVITABLES Y PÉRDIDA DE PRODUCTIVIDAD POR PM 2.5 , SEGÚN LA NORMA INTERNACIONAL

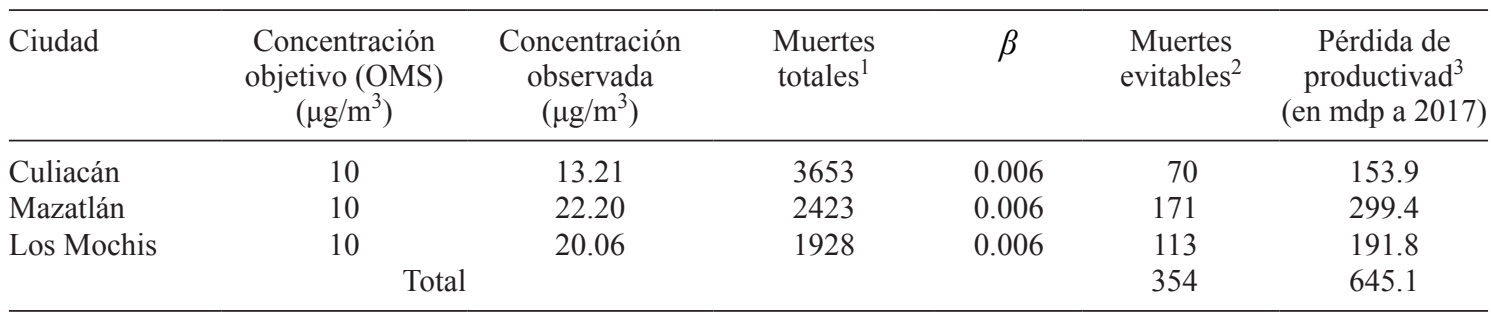

${ }^{1}$ Excluyen las accidentales y violentas; ${ }^{2}$ si se redujera la emisión de $\mathrm{PM}_{2.5}$ de la concentración observada a la concentración objetivo; ${ }^{3}$ por muertes asociadas a la emisión de $\mathrm{PM}_{2.5}$

Fuente: elaboración propia

CUADRO VII. MUERTES EVITABLES Y PÉRDIDA DE PRODUCTIVIDAD POR PM 2.5 , SEGÚN NORMA MEXICANA

\begin{tabular}{lccccrr}
\hline Ciudad & $\begin{array}{c}\text { Concentración objetivo } \\
(\mathrm{NOM}-025-S S A 1-2014) \\
\left(\mu \mathrm{g} / \mathrm{m}^{3}\right)\end{array}$ & $\begin{array}{c}\text { Concentración } \\
\text { observada }\left(\mu \mathrm{g} / \mathrm{m}^{3}\right)\end{array}$ & $\begin{array}{c}\text { Muertes } \\
\text { totales }\end{array}$ & $\beta$ & $\begin{array}{c}\text { Muertes } \\
\text { evitables }^{2}\end{array}$ & $\begin{array}{c}\text { Pérdida de productivad }^{3} \\
(\text { en mdp a 2017) }\end{array}$ \\
\hline Culiacán & 12 & 13.21 & 3653 & 0.006 & 26 & 58.3 \\
Mazatlán & 12 & 22.20 & 2423 & 0.006 & 144 & 251.8 \\
Los Mochis & 12 & 20.06 & 1928 & 0.006 & 91 & 154.6 \\
& & & & & 261 & 464.7 \\
\hline
\end{tabular}

${ }^{1}$ Excluyen las accidentales y violentas; ${ }^{2}$ si se redujera la emisión de $\mathrm{PM}_{2.5}$ de la concentración observada a la concentración objetivo; ${ }^{3}$ por muertes asociadas a la emisión de $\mathrm{PM}_{2.5}$ Fuente: elaboración propia 
De acuerdo con los daos de los cuadros VI y VII, el gobierno de Sinaloa se ahorraría una cantidad positiva y medible de recursos económicos si actúa para mitigar el problema de la contaminación del aire por $\mathrm{PM}_{2.5}$. Se podría decir que el costo económico por exceder los límites permisibles de $\mathrm{PM}_{2.5}$ en las principales ciudades de Sinaloa está en un rango de 24 a 34 millones de dólares por año debido a la pérdida de productividad en la población considerada para este estudio.

Por lo anterior, es recomendable diseñar e implementar políticas locales que promuevan la reducción de los niveles de $\mathrm{PM}_{2.5}$ en las principales ciudades de Sinaloa. Identificar a los sectores que generan las emisiones de PM por tipo de fuente y contaminante es un asunto de interés público que debe empezar a plantearse desde la óptica de los gobiernos locales. $\mathrm{Al}$ respecto, los avances a nivel internacional indican que las fuentes fijas son más fáciles de identificar y gestionar; no obstante, las fuentes móviles, principalmente los automóviles, pueden contribuir con reducciones sustanciales en las emisiones si se implementan programas específicos tendientes a regular este sector.

Debemos acotar que los resultados obtenidos evalúan las muertes evitables al reducir la contaminación a los niveles normativos contabilizando únicamente los impactos en la salud de $\mathrm{PM}_{2.5}$, sin incluir otros contaminantes igual o más dañinos que son emitidos al ambiente. Además, otros aspectos han quedado fuera de este análisis, como los gastos por hospitalización, días no laborados por enfermedad y efectos de la exposición a largo plazo a partículas, entre otros. También distintos enfoques y herramientas deberán ser explorados para confirmar y/o mejorar estos resultados.

Finalmente, es urgente reducir la exposición de personas a partículas potencialmente dañinas, que a medida que rebasan ciertos límites aumentan el riego de afectación, lo cual incluso puede desembocar en la muerte. Aparte de evitar un costo económico a la sociedad en general y a una familia en particular, cada fallecimiento que se evita gracias al descenso de la contaminación es un logro que enaltece al ser humano.

\section{CONCLUSIONES}

Siguiendo la metodología de evaluación del impacto en la salud por emisión de partículas $\mathrm{PM}_{2.5} \mathrm{y}$ usando una herramienta desarrollada por el Instituto Mexicano de Competitividad (IMCO), se realizó un análisis empírico para las principales ciudades de
Sinaloa, México. Las ciudades de Culiacán (capital), Mazatlán y Los Mochis, Ahome, fueron las tres zonas urbanas analizadas. Los resultados encontrados demuestran que la concentración promedio anual de este contaminante para 2017 fue superior a los límites máximos recomendados por la norma internacional de la OMS y la Norma Oficial Mexicana NOM025-SSA1-2014. También se estimaron las muertes prematuras evitables si la concentración promedio de $\mathrm{PM}_{2.5}$ se redujera a los límites indicados. Se encontró que las muertes totales evitables serían 354 si cumpliera con la normativa de la OMS y 261 con la norma mexicana, respectivamente.

Otro resultado alcanzado en este estudio fue calcular el impacto económico de las muertes asociadas a la exposición de la población a $\mathrm{PM}_{2.5}$. Para ello se utilizaron métodos que contabilizan las pérdidas de productividad ocasionadas por dichas muertes, los cuales fueron explicados en el apartado metodológico. Cuando el ejercicio se realizó con el parámetro de la norma internacional, se estimó un costo económico de 645.1 millones de pesos mexicanos, equivalente a 34.1 millones de dólares estadounidenses para 2017. Dado que la norma mexicana es más laxa, cuando se estimaron los impactos de la contaminación por $\mathrm{PM}_{2.5}$ en la salud, los resultados fueron menores, traduciéndose en un costo de 464.7 millones de pesos, equivalente a 24.6 millones de dólares estadounidenses para el mismo año.

Se concluye que la ciudad más contaminada y con mayores impactos en la salud por efecto de la exposición a este contaminante es Mazatlán y la menos afectada es Culiacán. No obstante, la población de las tres ciudades está expuesta a niveles de $\mathrm{PM}_{2.5}$ superiores a los permisibles por la normatividad nacional e internacional, por lo que existe un riesgo real de que la población de esas ciudades pueda morir por exposición al contaminante estudiado.

Por último, se recomienda que las autoridades locales diseñen e implementen medidas que ayuden a disminuir los niveles de contaminación por $\mathrm{PM}_{2.5}$ con el objetivo de evitar, en primer lugar, las muertes prematuras, $\mathrm{y}$, en segundo lugar, la reducción de las capacidades productivas de la región. Esto significaría un beneficio económico para el gobierno, dado el menor gasto requerido, y para la sociedad un incremento en el bienestar.

\section{REFERENCIAS}

Alpízar F., Piaggo M. y Pacay E. (2017). Valoración económica de los beneficios en la salud asociados a 
la reducción de la contaminación del aire. El caso de la Gran Área Metropolitana de Costa Rica. Comisión Económica para América Latina y el Caribe. Santiago de Chile, Chile, 59 pp.

Apte J.S., Brauer M., Cohen A.J., Ezzati M. y Pope III A.C. (2018). Ambient $\mathrm{PM}_{2.5}$ reduces global and regional life expectancy. Environ. Sci. Tech. Let. 5 (9), 546-551. DOI: 10.1021/acs.estlett.8b00360

BANXICO (2018). Relación peso-dólar. Banco de México [en línea]. http://www.banxico.org.mx/SieInternet/ consultarDirectorioInternetAction. . ? sector $=6 \& \mathrm{idC}$ uadro $=$ CF $373 \&$ accion $=$ consultarCuadro $\&$ locale $=$ es 24/09/18.

Chanel O., Pérez P., Künzli K., Medina S. y Aphekom group. (2016). The hidden economic burden of air pollution-related morbidity: Evidence from the Aphekom project. Eur. J. Health. Econ. 17 (9), 1101-1015.

DOI: $10.1007 / \mathrm{s} 10198-015-0748-\mathrm{z}$

Chang T., Ziving J.G., Gross T. y Neidell M. (2016). Particulate pollution and the productivity of pear packers. Am. Econ. J.-Econ. Polic. 8 (3), 141-169. DOI: $10.1257 /$ pol.20150085

Cohen A.J., Anderson H.R., Ostro B., Pandey K.D., Krzyzanowski M., Künzli N., Gutschmidt K., Pope III C.A., Romieu I., Samet J.M. y Smith K. (2005). The global burden of disease due to outdoor air pollution. J. Toxicol. Env. Heal A. 68 (13-14), 1301-1307. DOI: $10.1080 / 15287390590936166$

Cohen A.J., Brauer M., Burnett R., Anderson H.R., Frostad J., Estep K., Balakrishnan K., Brunekreef B., Dandona L., Dandona R., Feigin V., Freedman G., Hubbell B., Jobling A., Kan H., Knibbs L., Liu Y., Martin R., Morawska L., Pope C.A. 3rd., Shin H., Straif K., Shaddick G., Thomas M., van Dingenen R., van Donkelaar A., Vos T., Murray C.J.L. y Forouzanfar M.H. (2017). Estimates and 25-year trends of the global burden of disease attributable to ambient air pollution: an analysis of data from the Global Burden of Diseases Study 2015. Lancet 389 (10082), 1907-1918.

DOI: $10.1016 / \mathrm{S} 0140-6736(17) 30505-6$

CONAPO (2017) Proyección de la población de México y de las Entidades Federativas, 2016-2050. Consejo Nacional de Población, México. https://datos.gob.mx/bus$\mathrm{ca} /$ dataset/proyecciones-de-la-poblacion-de-mexico-yde-las-entidades-federativas-2016-2050-16/09/2018

De Keijzer C., Agis D., Ambrós A., Arévalo G., Baldasano J.M., Bande S., Barrera-Gómez J., Benach J., Cirach M., Dadvand P., Ghigo S., Martinez-Solanas Ė., Nieuwenhuijsen M., Cadum E. y Basagaña X. (2017). The association of air pollution and greenness with mortality and life expectancy in Spain: A small-area study. Environ. Int. 99, 170-176.

DOI: $10.1016 /$ j.envint.2016.11.009
Dockery D.W., Pope C.A., Xu X., Splengler J.D., Ware J.H., Fay M.E., Ferris B.G. Jr. y Speizer F.E. (1993). An association between air pollution and mortality in six US Cities. N. Engl. J. Med. 329 (24), 1753-1759. DOI: 10.1056/NEJM199312093292401

Dockery D.W. y Pope C.A. (1994). Acute respiratory effects of particulate air pollution. Environmental Epidemiology Program. Harvard School of Public Health, Boston, Massachusetts. Annu. Rev. Publ. Health. 15, 107-132.

DOI: 10.1146/annurev.pu.15.050194.000543

Evans J., van Donkelaar A., Martin R.V., Burnett R., Rainham D.G., Birkett N.J. y Krewski D. (2013). Estimates of global mortality attributable to particulate air pollution using satellite imagery. Environ. Res. 120, 33-42.

DOI: 10.1016/j. envres.2012.08.005

Forouzanfar M.H., Burnett R., Cohen A., Abera S.F., Bell M.L., Brunekreef B., Chen H., Dicker D., Eshrati B., Franklin R.C., Gakidou E., Gupta R., Hoek H.W., Hubbell B.J., Ismayilova S., Jensen P.N., Kwan G.F., Lozano R., Mueller U.O., Nieuwenhuijsen M.J., Nyakarahuka L., Orozco R., Pope C.A., Quezada A.D., Romieu I., Stein D.J., Texcalac-Sangrador J.L., Uzun S.B., Van Dingenen R., Wright J.L., Younoussi Z., Zheng Y. y Zunt J.R. (2015). Global, regional, and national comparative risk assessment of 79 behavioural, environmental and occupational, and metabolic risks or clusters of risks in 188 countries, 1990-2013: A systematic analysis for the Global Burden of Disease Study 2013. Lancet 386 (10010), 2287-2323. DOI: $10.1016 / \mathrm{S} 0140-6736(15) 00128-2$

Gakidou E., Afshin A., Abajobir A.A., Abate K.H., Abbafati C., Abbas K.M., Barquera S., Bell M.L., Bennett D.A., Brauer M., Cárdenas R., Chang H.Y., Cohen A.J., Cooper C., Dandona L., Dandona R., Donkelaar A.V., Ebrahimi H., Eshrati B., Farzadfar F., Foreman K.J., Furtado J.M., García-Basteiro A.L., Gupta R., Gupta T., Gupta V., Hoek H.W., Hu G., Huang J.J., Huang H., Ibrahim N.M., Ikeda C., Jacobsen K.H., Johansson L.R.K., Jürisson M., Kabir Z, Kadel R., Kemmer L., Kravchenko M., Kyu H.H., Lee P.H., Levi M., Levy T.S., Lim S.S., Lopez A.D., Matsushita K., Miller T.R., Musa K.I., Nguyen M., Noubiap J.J.N., Obermeyer C.M., Ogbo F.A., Oh I.H., Olivares P.R., Olsen H.E., Ortiz A., Popova S., Poulton RG, Qorbani M., Radfar A., Reynales-Shigematsu L.M., Rivera J.A., Rojas-Rueda D., Roman Y., Salomon J.A., Seedat S., Shi P., Smith M., Steiner C., Thomson A.J., Uthman O.A., van Boven J.F.M., Varughese S., Vos T., Woodbrook R., Xavier D., Xu G., Younis M.Z., y Zodpey S. (2017). Global, regional, and national comparative risk assessment of 84 behavioural, 
environmental and occupational, and metabolic risks or clusters of risks, 1990-2016: A systematic analysis for the Global Burden of Disease Study 2016. Lancet 390 (10100), 1345-1422.

DOI: 10.1016/S0140-6736(17)32366-8

Hoek G., Krishnan R.M., Beelen R., Peters A., Ostro B., Brunekreef B. y Kaufman J.D. (2013). Long-term air pollution exposure and cardio-respiratory mortality: A review. Environ. Health. 12 (1), 1-16.

DOI: $10.1186 / 1476-069 \mathrm{X}-12-43$

Hurley F., Alistair H., Hilary C.M., Brian M., Stephen P. y Watkiss P. (2005). Methodology for cost-benefit analysis for CAFE. Vol. 2. Health Impact Assessment. AEA Technology. Didcot, Oxon, UK, 133 pp.

IMCO (2013). ¿Cuánto nos cuesta la contaminación del aire en México? Impactos en salud y en productividad para 34 ciudades mexicanas. Anexo metodológico. Instituto Mexicano para la Competitividad [en línea]. https://imco.org.mx/wp-content/ uploads/2013/09/Anexo-Metodol\%C3\%B3gico24Sep13.pdf 16/09/18.

INE (2011). Guía para evaluar los impactos en la salud por la instrumentación de medidas de control de la contaminación atmosférica. Instituto Nacional de Ecología-Secretaría de Medio Ambiente y Recursos Naturales. Ciudad de México, México, 82 pp.

INECC (2014). Valoración económica de los beneficios a la salud de la población que se alcanzarían por la reducción de las $\mathrm{PM}_{2.5}$ en tres zonas metropolitanas mexicanas. Instituto Nacional de Ecología y Cambio Climático. Ciudad de México, México, 51 pp.

INEEC (2017). Estimación de impactos en salud por contaminación atmosférica en la región centro del país y alternativas de control. Instituto Nacional de Ecología y Cambio Climático. Ciudad de México, México, $73 \mathrm{pp}$.

INEGI (2010). Censo de Población y Vivienda 2010. Instituto Nacional de Estadística y Geografía, Aguascalientes, México [en línea].http://www.inegi.org. $\mathrm{mx} / \mathrm{sistemas/olap/Proyectos/bd/censos/cpv2010/}$ PT.asp?s=est\&c $=27770 \&$ proy $=\mathrm{cpv} 10$ pt 16/09/18.

INEGI (2015). Densidad de población (habitantes por kilómetro cuadrado). Instituto Nacional de Estadística y Geografía. Aguascalientes, México [en línea]. http:// www.beta.inegi.org.mx/app/buscador/default.html?q $=$ densidad + poblacion + de + culiacan\#tabMCcollapseIndicadores 16/09/18.

INEGI (2016). Encuesta Nacional de Ingresos y Gastos de los Hogares (ENIGH) 2016. Instituto Nacional de Estadística y Geografía. Aguascalientes, México, 39 pp.

INEGI (2017a). Anuario estadístico y geográfico de Sinaloa 2016. Instituto Nacional de Estadística y Geografía. Aguascalientes, México, 478 pp.
INEGI (2017b). Índice de precios al consumidor. Instituto Nacional de Estadística y Geografía. Aguascalientes, México, [en línea].https://www.inegi.org.mx/app/ tabulados/default.aspx?nc=ca55_2018 25/08/2018.

Krewski D., Jerrett M., Burnett R.T., Ma R., Hughes E., Shi Y., Turner M.C., Pope C.A. 3rd. Thurston G., Calle E.E., Thun M.J., Beckerman B., DeLuca P., Finkelstein N., Ito K., Moore D., Newbold K.B., Ramsay T., Ross Z., Shin H.H., y Tempalski B. (2009). Extended followup and spatial analysis of the American Cancer Society study linking particulate air pollution and mortality. Health Effects Institute. Boston, Massachusetts, EUA, $154 \mathrm{pp}$.

Lim S.S., Vos T., Flaxman A.D., Danaei G., Shibuya K., Adair-Rohani H., Amann M., Anderson H.R., Andrews K.G. y Aryee M., Atkinson C., Bell M.L., Brooks P., Brunekreef B., Burnett R.T., Chen H., Chen J.S., Cheng A.T., Child J.C., Cohen A., Des Jarlais D.C., Edmond K., Engell R.E., Ezzati M., Farzadfar F., Ferrari A., Freedman G, Freeman M.K., Gakidou E., Gunnell D., Gutierrez H.R., Hoek H.W., Hogan A., Hubbell B.J., Ibeanusi S.E., Jonas J.B., Kan H., Khoo J.P., Laden F., Li Y., London S., Lozano R., Lu Y., Mehta S., Micha R., Murphy T., Naghavi M., Neal B., Osborne R., Ostro B., Pope D, Pope C.A. 3rd., Roberts T., RodriguezPortales J.A., Romieu I., Salomon J.A., Shield K., Thurston G.D., Van Dingenen R., van Donkelaar A., Williams W., Wilson N., Yip P. y Zielinski J.M. (2012). A comparative risk assessment of burden of disease and injury attributable to 67 risk factors and risk factor clusters in 21 regions, 1990-2010: A systematic analysis for the Global Burden of Disease Study 2010. Lancet 380 (9859), 2224-2260.

DOI: $10.1016 / \mathrm{S} 0140-6736(12) 61766-8$

Morris J.A. y Gardner M.J. (1988). Calculating confidence intervals for relative risks (odds ratios) and standardised ratios and rates. Brit. Med. J. 296 (6632), 1313-1316. DOI: 10.1136/bmj.296.6632.1313

Naghavi M., Abajobir A.A., Abbafati C., Abbas K.M., et al. (2017). Global, regional, and national age-sex specific mortality for 264 causes of death, 1980-2016: A systematic analysis for the Global Burden of Disease Study 2016. Lancet 390 (10100), 1151-1210. DOI: $10.1016 / \mathrm{S} 0140-6736(17) 32152-9$

OMS (2001). Quantification of health effects of exposure to air pollution report on a WHO working group, Bilthoven, Netherlands, 20-22 November 2000. World Health Organization. Regional Office for Europe. Copenhague, Dinamarca, 29 pp.

OMS (2006). Guías de calidad del aire de la OMS relativas al material particulado, el ozono, el dióxido de nitrógeno y el dióxido de azufre. Organización Mundial de la Salud. Ginebra, Suiza, 25 pp. 
OMS (2016). Ambient air pollution: A global assessment of exposure and burden of disease. World Health Organization. Ginebra, Suiza, $121 \mathrm{pp}$.

Pascal M., Corso M., Chanel O., Declercq C., Badaloni C., Cesaroni G., Henschel S., Meister K., Haluza D., Martin-Olmedo P. y Medina S. (2013). Assessing the public health impacts of urban air pollution in 25 European cities: results of the Aphekom project. Sci. Total. Environ. 449, 390-400.

DOI: 10.1016/j.scitotenv.2013.01.077

Pope C.A. 3rd., Burnett R.T., Turner M.C., Cohen A., Krewski D., Jerrett M., Gapstur S.M. y Thun M.J. (2011). Lung cancer and cardiovascular disease mortality associated with ambient air pollution and cigarette smoke: shape of the exposure-response relationships. Environ. Health. Persp. 119 (11), 1616-1621. DOI:10.1289/ehp.1103639

Rice D.P., Hodgson T.A. y Kopstein A.N. (1985). The economic costs of illness: A replication and update. Health. Care. Financ. R. 7 (1), 61-80.

Rojas-Bracho L. y Garibay-Bravo V. (2003). Partículas suspendidas, aeropartículas o aerosoles, ¿hacen daño a la salud? ¿Qué hacer? Gaceta Ecológica del Instituto Nacional de Ecología 69, 29-44.
SAGARPA (2017). Sinaloa. Infografía Alimentaria. Servicio de Información Agroalimentaria y Pesquera (SIAP), Secretaría de Agricultura, Ganadería, Desarrollo Rural, Pesca y Alimentación [en línea]. https:// www.gob.mx/siap/articulos/infografias-agroalimentarias-2016-101148 25/08/2019.

SEMARNAT (2012). Norma Oficial Mexicana NOM156-SEMARNAT-2012. Establecimiento y operación de sistemas de monitoreo de la calidad del aire. Secretaría de Medio Ambiente y Recursos Naturales. Diario Oficial de la Federación, 16 de julio.

SSA (2014). Norma Oficial Mexicana NOM-025SSA1-2014. Salud ambiental. Valores límite permisible para la concentración de partículas suspendidas $\mathrm{PM}_{10}$ y $\mathrm{PM}_{2.5}$ en el aire ambiente y criterios para su evaluación. Secretaría de Salud. Diario Oficial de la Federación, 20 de agosto.

USEPA (2011). The benefits and costs of the Clean Air Act: 1990 to 2020. Final report. Office of Air and Radiation, United States Environmental Protection Agency. Washington, D.C., EUA, 238 pp.

Wu R., Dai H., Geng Y., Xie Y., Masui T., Liu Z. y Qian Y. (2017). Economic impacts from $\mathrm{PM}_{2.5}$ pollutionrelated health effects: A case study in Shanghai. Environ. Sci. Technol. 51 (9), 5035-5042.

DOI: $10.1021 /$ acs.est. $7 b 00026$ 